\title{
The EuroSprite2005 Observational Campaign: an example of training and outreach opportunities for CAL young scientists
}

\author{
O. Chanrion ${ }^{1, *}$, N. B. Crosby ${ }^{2}$, E. Arnone ${ }^{3, *}$, F. Boberg ${ }^{3, *}$, O. Van der Velde ${ }^{4,}{ }^{*}$, A. Odzimek $^{3, *}$, Á. Mika $^{5, *}$, \\ C.-F. Enell ${ }^{6, *}$, P. Berg ${ }^{7, *}$, M. Ignaccolo ${ }^{8, *}$, R. J. Steiner ${ }^{5, *}$, S. Laursen ${ }^{1}$, and T. Neubert ${ }^{1}$ \\ ${ }^{1}$ Danish National Space Center, Technical University of Denmark, Copenhagen, Denmark \\ ${ }^{2}$ Belgian Institute for Space Aeronomy, Brussels, Belgium \\ ${ }^{3}$ Department of Physics and Astronomy, University of Leicester, Leicester, UK \\ ${ }^{4}$ Laboratoire d'Aérologie, Paul Sabatier University, Toulouse, France \\ ${ }^{5}$ Department of Physics, University of Crete, Heraklion, Crete, Greece \\ ${ }^{6}$ Sodankylä Geophysical Observatory, University of Oulu, Sodankylä, Finland \\ ${ }^{7}$ Danish Meteorological Institute, Copenhagen, Denmark \\ ${ }^{8}$ Department of Electronic and Electrical Engineering, University of Bath, Bath, UK \\ ${ }^{*}$ CAL Young Scientist
}

Received: 14 November 2006 - Revised: 15 February 2007 - Accepted: 8 March 2007 - Published: 30 July 2007

\begin{abstract}
The four year "Coupling of Atmospheric Layers (CAL)" EU FP5 Research Training Network project studied unanswered questions related to transient luminous events (sprites, jets and elves) in the upper atmosphere. Consisting of ten scientific work-packages CAL also included intensive training and outreach programmes for the young scientists hired. Educational activities were based on the following elements: national $\mathrm{PhD}$ programmes, activities at $\mathrm{CAL}$ and other meetings, a dedicated summer school, and two European sprite observational campaigns. The young scientists were strongly involved in the latter and, as an example, the "EuroSprite2005" observational campaign is presented in detail. Some of the young scientists participated in the instrument set-up, others in the campaign logistics, some coordinated the observations, and others gathered the results to build a catalogue. During the four-month duration of this campaign, all of them took turns in operating the system and making their own night observations. The ongoing campaign activities were constantly advertised and communicated via an Internet blog. In summary the campaign required all the CAL young scientists to embark on experimental work, to develop their organisational skills, and to enhance their ability to communicate their activities. The campaign was a unique opportunity to train and strengthen skills that will be an asset to their future careers and, overall, was most successful.
\end{abstract}

Correspondence to: O. Chanrion

(chanrion@spacecenter.dk)

\section{Introduction}

Sprites, jets and elves are "celestial fireworks" observed above particular thunderclouds. They are also referred to as "transient luminous events (TLEs)", sprites specifically as "high altitude lightning". TLEs occur mainly above large continental thunderstorms, for example over the Great Plains of the United States, where they were first observed in 1989. They have been observed from ground based observation platforms in South America, Africa and southern Europe, and also from space borne observational platforms. Sprites are the result of quasi-electrostatic fields, jets of predischarge thundercloud fields and elves of the electromagnetic pulse radiated by the lightning return stroke (see Fig. 1).

In November 2002 the four-year Coupling of Atmospheric Layers (CAL) research training network was funded by the European Commission within the Marie Curie Actions to study unanswered questions relating to TLEs and their effects on the atmosphere. More specifically, CAL concerned thunderstorms, electrical and space radiation effects in the stratosphere, mesosphere and lower thermosphere. The research was also aimed at studying the relation of TLEs with various aspects of the atmospheric system and thus climate (Neubert, 2003; Arnold et al., 2003).

The aim of EU research training networks is to allow young scientists at the start of a scientific career to work in another country by integrating them into an international research project. Through the network they obtain training and research experience of high quality in an international working environment. Under the coordination of the Danish National Space Center (DNSC), CAL consisted of scientists (senior and young) from ten European institutes. During the

Published by Copernicus Publications on behalf of the European Geosciences Union. 


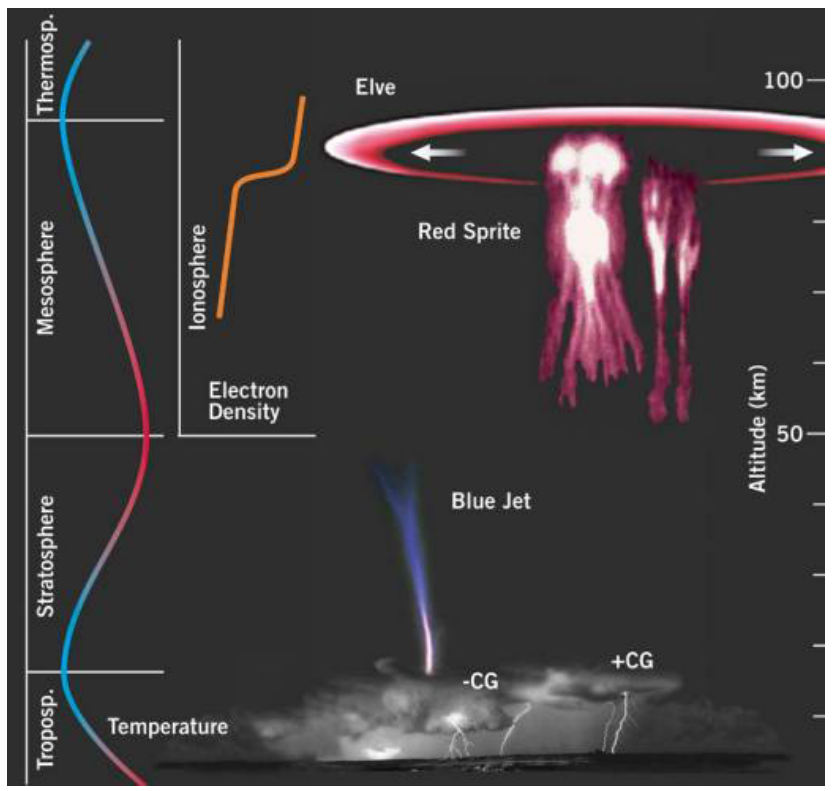

Fig. 1. Diagram illustrating the range of transient luminous events in the atmosphere. Reprinted with permission of Science (Neubert, 2003).

four years the CAL network hired twelve young European scientists, of which six were PhD students (pre-docs) and six were postdoctoral scientists. The projected plan for the network regarding months of pre-docs vs. post-docs was modified due to problems of identifying suitable candidates. This stemmed from one of the EU requirements that the young scientist is from another country than the country of the host institution. In certain cases, it was only possible to find a suitable pre-doc, where a post-doc had been planned, and vice versa. However, in the end the fifty-fifty percent representation as well as the diverse nationalities (nine in all) provided a good balance of young scientists.

CAL consisted of ten scientific work-packages and two work-packages dedicated to the training and outreach aspects of the project. Spectacular images of high-altitude discharges make the subject of the CAL network ideal as outreach material for the general public. Activities of the CAL outreach programme included popular/semi-popular articles, press releases, interviews, an educational web site (http://www.eurosprite.net built in three levels: "beginners", "intermediate" and "advanced"), and a public CAL web site (servicing the network teams and the public). As part of their CAL work, all CAL young scientists were required to produce educational material concerning their research for middle school levels and above. As a result each young scientist obtained experience in constructing a web site, writing an "outreach" article, and giving a presentation to the general public and in schools. The young scientists received guidance and feed-back from the CAL senior scientist responsible for the outreach programme.
The educational "training" programme work-package was a key ingredient in the CAL project and covered the implementation of the training activities for the ten young scientists. The objectives included educating the young scientists:

- in a broad range of atmospheric disciplines,

- in a range of scientific methods,

- to collaborate across disciplinary boundaries,

- to coordinate and implement larger collaborations,

- to communicate scientific results to the public.

To accomplish these objectives, the young scientists of the network were involved in several activities. They attended several conferences, workshops and courses. They wrote webpages, they communicated their work to others, and they were encouraged to work together. The writing of educational web-pages as well as giving talks aimed at different audiences has further trained the young scientists to become better communicators. All young scientists attended the NATO ASI summer-school on "Sprites, Elves and Intense Lightning Discharges" held in Corte, Corsica, during the summer of 2004, as part of their training. The "summerschool" was organised by CAL network members and was co-sponsored by the CAL network. The themes of sprite research were classified in four categories: (1.) Meteorology and intense lightning discharges needed to produce sprites, elves and blue jets; (2.) Optical observations of TLEs; (3.) Electromagnetic radiation of intense lightning discharges and sprites; (4.) Theory and modelling in the areas (1-3). The programme included thematic lectures, tutorials given by the leaders in the field (group work discussions), experimental work (operation of optical cameras -sprite imaging) and presentation of research studies by participants, including the young scientists (poster evening). The format of the school gave the young scientists an opportunity to discuss their work directly with the senior scientists attending the school.

A central element of the training activity was the annual observational campaigns in Southern Europe during the summer months. During the second part of the CAL project two sprite observational campaigns "EuroSprite2005" [19 July 2005 to 15 November 2005] and "EuroSprite2006" [1 July 2006 to 18 November 2006] were organised and implemented mostly by the CAL young scientists with little assistance from the senior scientists as part of their training. In 2005 optical cameras were installed at the Observatory of Pic du Midi in the French Pyrénées and Puy de Dôme in the Massif Central in France. In 2006 optical cameras were also installed at the Observatory of Pic du Midi. Furthermore members of the Toulouse team performed optical sprite observations from the roof of the meteorological observatory of Mont Aigoual during 2006. During both observational campaigns the young scientists coordinated and operated the systems. Information and daily 
Table 1. Hardware details for the two camera systems.

\begin{tabular}{ll}
\hline & System hardware \\
\hline Puy de Dôme & Two low-light CCD cameras with S-Video output and serial RS232 control interface. \\
& Motorised platform with a serial RS232 interface. \\
& GPS unit with a serial RS232 interface. \\
& Personal computer with BT878 frame grabbers, hard drives and network interface cards. \\
& Remote controlled power switch with TCP/IP control interface. \\
& Two low-light CCD cameras with Camera Link digital output and control interface. \\
Pic du Midi & Photometer with a high speed serial RS485 interface. \\
& Motorised platform with a serial RS232 interface. \\
& GPS unit with a serial RS232 interface. \\
& Personal computer with Camera Link frame grabbers, hard drives and network interface cards. \\
& Remote controlled power switch with TCP/IP control interface. \\
& Several sensors (temperature, pressure, humidity) with serial RS232 interface.
\end{tabular}

updates were made available at the blog (http://eurosprite. blogspot.com) set up specially for this purpose.

By describing the "EuroSprite2005" observational campaign in more detail, this paper emphasises the involvement of the CAL young scientists in these observational campaigns and the opportunities which they created to strengthen skills useful for their scientist's future careers. Section 2 presents the experimental details of the two camera systems used in the "EuroSprite2005" campaign. The campaign (setting up the equipment, campaign organisation and communication) is presented in Sect. 3 and examples of the EuroSprite2005 observations are given in the next section. The CAL training evaluation is given along with the conclusions in Sect. 5.

\section{The experimental set-up}

The Eurosprite2005 observational campaign was a coordinated effort of the entire CAL network. Its main objective was to detect transient luminous events above active thunderstorms using ground-based observing facilities. Based on the $\mathrm{PhD}$ work of Thomas Allin (Allin, 2005; Allin et al., 2006) and the experience of the DNSC, two semi-automatic video observation facilities were installed in France. One system was located at the Puy de Dôme in the French Massif Central and the other at the Pic du Midi in the French Pyrénées. The two optical video camera systems include trigger software for automated optical event detection and were designed to be remotely controlled via the Internet. A main server located at the DNSC acted as a link between both camera systems and the operators and was used to store the data.

\subsection{The Puy de Dôme camera system}

The Puy de Dôme system consisted of two JAI (CVS3200) cameras, each fitted with a Sony ICX249AL $1 / 2^{\prime}$

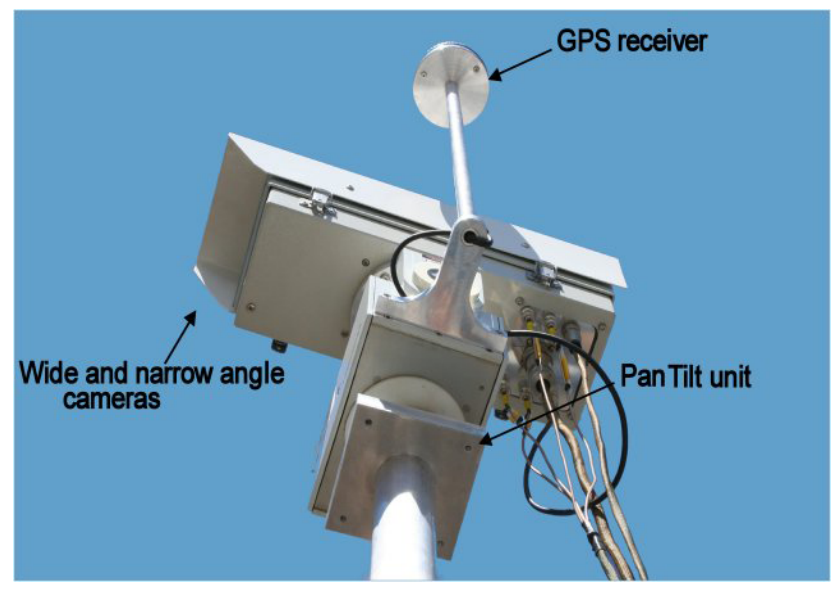

Fig. 2. The Puy de Dôme camera system.

monochrome ExView hole-accumulation diode (HAD) sensor. One camera was equipped with a $16 \mathrm{~mm}$ F1.4 lens for event detection and recording, and the other with a $25 \mathrm{~mm}$ F0.95 lens for high-resolution event recording. The cameras were mounted in weather proof housing on top of a QuickSet 20 motorised Pan-Tilt unit which allows for the pointing of the cameras within 360 degrees of azimuth and from -35 to +35 degrees of elevation (see Fig. 2). A GPS unit, providing both NMEA (National Marine Electronics Association) standard data and 1-pulse-per-second (PPS) signal, was used to ensure high precision time stamping of the data. A personal computer was used to control the instruments and record the data. A summary of the system hardware is listed in Table 1.

The PC operating system was based on a Linux distribution patched to support nano-precision timing, 1-PPS signal and the Video 4 Linux II interface layer. Several tools were installed to control the instruments and handle the data: 


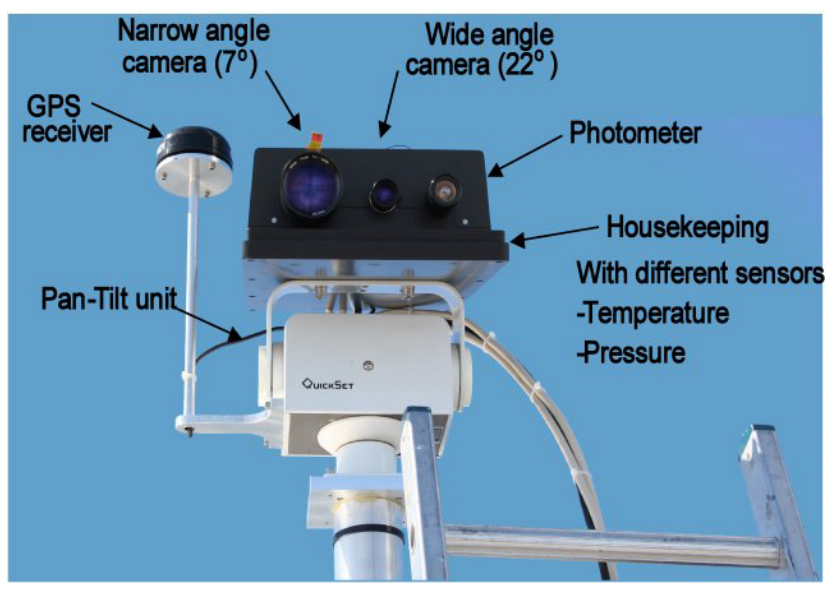

Fig. 3. The Pic du Midi camera system.

- camera control software to control the camera settings,

- guiding software to pilot the motorised platform,

- event detection software to analyse the images from the cameras and to store the pictures whenever an event occurs,

- several scripts to upload control information and data to the main server located in Denmark.

\subsection{The Pic du Midi camera system}

The Pic du Midi system was, with a few exceptions, similar to the system located at Puy de Dôme. It contained a photometer and the two cameras were of higher resolution (JAI CV-M4 ${ }^{+}$cameras fitted with a Sony ICX285AL 2/3' monochrome ExView HAD sensor). One was equipped with a $16 \mathrm{~mm}$ F1.4 lens for event detection and recording, and the other with a $50 \mathrm{~mm}$ F0.95 lens for high-resolution recording. The housing hosted heaters and different sensors to measure temperature, pressure and humidity in the internal environment. Table 1 lists the system hardware and Fig. 3 shows the camera system.

The PC operating system was identical to the one at Puy de Dôme. Most of the software was similar although some of it needed to be adapted to the individual instruments. New software tools were installed to:

- control and acquire data from the photometer,

- read sensor measurements.

\subsection{The control and storage server}

The DNSC hosted the control and storage server of the observational system. It was used to:

- filter the IP addresses that were allowed to connect to the camera systems,
- host a control web page,

- download and store the data from the camera systems.

In order for the young scientists to be able to operate the system from the country where they were working, the system was designed to be controlled remotely via the Internet. To operate a camera system, the first task was to connect to the control server. From this server they could control the power of the instruments, logon to the PCs, set-up the instrument and launch the event detection software.

This server hosted a control webpage (see Fig. 4) to help the operation of the camera system. For both observation stations, a webpage displayed in real-time control and/or triggered images from the cameras, lightning activity over Western Europe from the French lightning detection network Météorage (http://www.meteorage.com) and the cloud cover Meteosat image from the University of Ulm (http://meteosat. e-technik.uni-ulm.de). This server was also used to store the data in order to make them available to other members of the CAL network.

\section{The campaign progress}

\subsection{Camera system set-up}

The hardware was built and mounted by the engineers of the DNSC. From the early beginning of system preparation in the laboratory to the final installation on top of the mountains, young scientist Olivier Chanrion was integrated in the team and collaborated by performing software installation and camera set-up. The Puy de Dôme system was similar to the one used by Thomas Allin in 2003 and 2004 (Neubert et al., 2005); thus, only installation and set-up were needed. On the other hand, the Pic du Midi system required a new camera control and event-detection software, rewritten by the young scientist for the campaign. For the first time in his career Olivier Chanrion was involved in such an instrument setup in which he took the system administration responsibility. This gave him the opportunity to strengthen his knowledge in software development for hardware and acquire training in designing and documenting a system to be used by others.

\subsection{Organisation}

After the set-up, the young scientists operated the cameras without any help from the senior scientists. The young scientist Enrico Arnone took the lead in coordinating the observations. Before the campaign started he helped all the young scientists to define their campaign tasks and schedule, gathered all the organisational information on a coordination webpage, and set up an electronic mailing list to facilitate communication between observers.

E. Arnone performed the first two weeks of observations together with O. Chanrion and young scientist Á. Mika. 


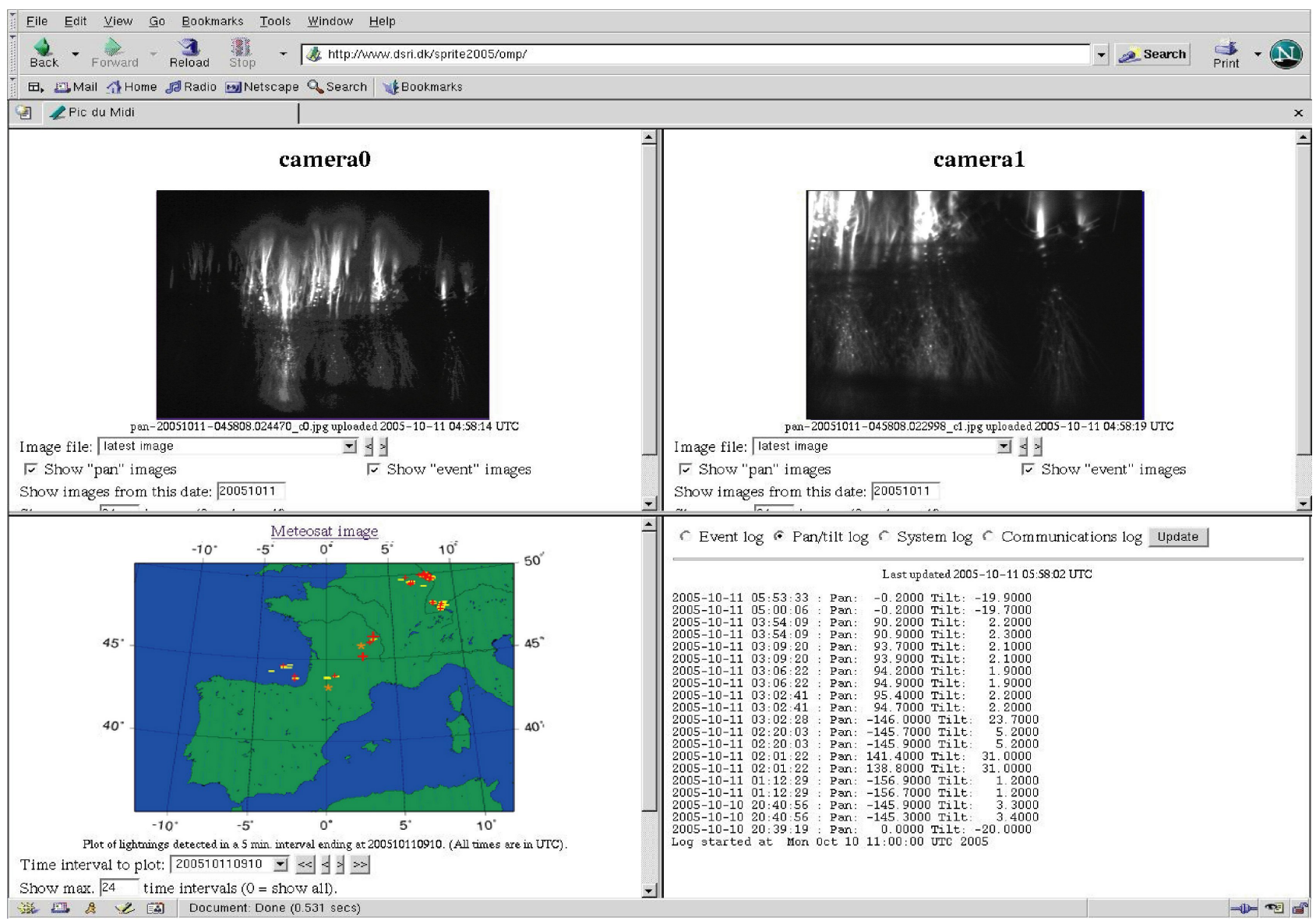

Fig. 4. The two top panels of the control webpage show control (left) and triggered (right) images from the cameras. The bottom left panel shows lightning activity or Meteosat images, and the bottom right panel shows log files from the system.

During these weeks, the three young scientists interacted strongly to check and finalise the system installation. To make the system as easy as possible to use, Á. Mika wrote a tool to compute the Pan-Tilt values from the geographical coordinates of the thunderstorm to be viewed and O. Chanrion wrote a tutorial explaining how the system was to be operated. This finalised the starting stage and the system was fully operational with instructions described in the tutorialmanual.

The campaign ran from mid-July to mid-November 2005 and during this period the ten young scientists took turns operating the system. Every week two young scientists were in charge of operating one camera system each. Each night, according to the weather conditions and the opportunity to catch an event, the young scientists decided whether to operate or not. The two operators interacted strongly, one informing the other about the storm to be observed. This synchronisation was necessary to allow the same sprite to be captured from both locations and thus to be able to calculate precisely its position and dimension. In order to help the decision of when and what to observe, and to avoid missing the oppor- tunity to catch an interesting event, the young scientist Oscar Van der Velde published periodically a weather forecast, in which he stressed thunderstorm activities which promised sprites.

The campaign created an excellent environment for collaboration between the young scientists. Using an electronic mailing list, the scientists discussed and exchanged new ideas. Many interesting discussions took place, some aimed at improving the system and the making of observations; others were comments or congratulations for the first sprite results. By putting the entire responsibility for operating the system in the hands of the young scientists, all of them were obliged to organise themselves carefully and to work as a team to fulfil a common goal.

\subsection{Simultaneous non-optical observations}

Since sprites can also be detected by non-optical observations, several instruments had been deployed over Europe that recorded data simultaneously. While the optical observations ran, some of the young scientists of the network participated in such observations too. 

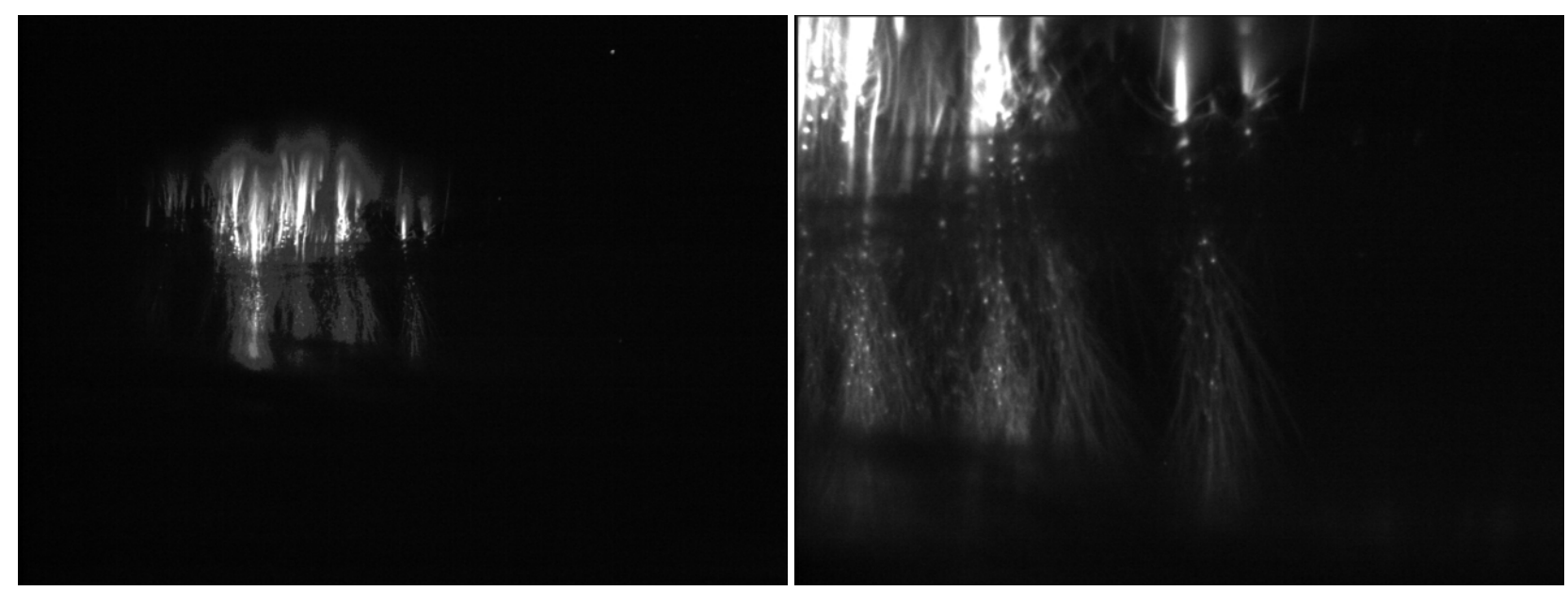

Fig. 5. A sprite observed by the young scientist Á. Mika on 29 July at 01:40:50 UT. The two panels show sprite images from the wide angle camera (left) and the narrow angle camera (right) at Pic du Midi.

An Extremly, Ultra and Very Low Frequency (ELF-ULFVLF) radio receiver, able to record weak electromagnetic signal perturbations, was operated on a campaign basis by the Sodankylä Geophysical Observatory in Finland. The young scientist Carl-Fredrik Enell helped with the antenna installation.

The young scientists Á. Mika and R. J. Steiner were involved in the set-up and operation of the two VLF radio receivers that were installed at the University of Crete.

Young scientist A. Odzimek crosschecked the timestamping accuracy of both camera systems. She compared the optical observations, ELF data from the Schumann Resonance Laboratory in Poland and lightning data from Météorage.

\subsection{Communication of the campaign activities}

During the campaign "The Sprite2005 Observation Campaign" Internet Blog was created and made accessible through http://eurosprite.blogspot.com. O. Van der Velde and E. Arnone maintained this blog. All the important progress of the system set-up, weather forecast and "sprite catches" were posted on the blog by all young scientists. This blog was visited by a wide international community interested in sprites.

Results from the campaign were presented by the young scientists themselves during meetings and international conferences. Especially, the campaign was highlighted by Olivier Chanrion and Enrico Arnone during an exhibition at the "Communicating European Research (CER) 2005" International Conference in Belgium where the objective was to show the CAL network training and outreach activities. In addition $\mathrm{O}$. Chanrion was interviewed by a French journalist about the campaign that is mentioned in the June 2006 edition of the popular scientific magazine "Ciel et Espace".
The young scientists had the opportunity to strengthen their capabilities in communicating their research to several types of audiences. They learned to report their observations in a systematic way for future use, to present their work at international conferences and also to communicate their activities to a non-specialised public.

\section{Observations}

The EuroSprite2005 observational campaign ran over about 120 nights; during this period 64 images of sprites were recorded during 19 storms. These optical recordings were complemented by infrasound, electromagnetic (in the ultra low, extremely low and very low frequency ranges) and lightning location recordings. An example of a sprite recorded at the Pic du Midi is seen in Fig. 5 in normal mode (left panel) and high resolution (right panel).

In order to help the analysis of the results from the campaign, the young scientist P. Berg designed a form that every operator had to fill in after each night of observation and the young scientist $F$. Boberg gathered all the results in a web database. For all events observed, this database gives the time of the event, the position and return stroke current of the associated lightning, links to the images from the cameras, and links to the corresponding lightning and Meteosat map. This webpage is a useful tool for any person wanting to exploit the dataset.

At present, some of the young scientists are using this catalogue to study the one-to-one relationship between sprite occurrence and very low frequency radio transients. Moreover, the EuroSprite2005 observational campaign will provide a better accuracy for the detection efficiency and false alarm rate of sprite detectors based on non-optical recordings. 
Thus, a more accurate estimation of the rate of sprite occurrences on Earth will be possible.

\section{Conclusions}

The CAL research training network project ended in October 2006. The European flavour of CAL was the participation of ten young scientists from a wide-range of European countries. Moving abroad, international collaborations, interdisciplinary scientific work (theoretical and practical), and new cultural experiences, applied to all the CAL young scientists.

The evaluation of the main training objectives of the CAL project listed in Sect. 1 has been interactive and continuous. No specific formula was used to assess the progress. Instead both senior and young scientists have systematically exchanged impressions among themselves and yearly reports containing these were written (input to the yearly CAL reports). Because of the cross-disciplinary nature of the CAL subject matter, the young scientists have been connected with groups and topics that were new to them, allowing them to apply their areas of expertise to new problems. Thus they have learned to collaborate across disciplinary boundaries. They have been educated in a broad range of atmospheric disciplines and in a diverse range of scientific methods. In parallel they also learned to communicate scientific results to the public; they were enthusiastic about outreach activities in general.

During the CAL project the young scientists learned to coordinate and implement larger collaborations. Especially, they were successfully brought into the organisation and management of workshops/yearly meetings and the experimental campaigns. The "EuroSprite2005" and "EuroSprite2006" observational campaigns were organised by the CAL research training network and in this paper we have presented the "EuroSprite2005" campaign in detail. This successful campaign provided an experimental training opportunity for the CAL young scientists. They enhanced their organisational skills by sharing tasks in the preparation of the instruments and the observation schedule, by relieving one another during the four months and by performing their own sprite observations. They strengthened their communication skills by advertising the campaign on an Internet blog and by presenting the results from the campaign both to fellow scientists and to the general public. This type of activity is a unique way for young scientists to obtain experience when working together as a team, an asset that will benefit them in their future scientific careers. As one of the CAL young scientists stated in regard to the EuroSprite observational campaigns: "Hands-on scientific work forces people to communicate and solve problems as a group" (Crosby, 2006).

During the annual meetings all CAL participants (young and old) had an opportunity to reflect on the past year and provide feed-back to the network as input for "lessons learned". If there was a problem the goal was to find a solu- tion. In summary the CAL network has been of great value to all participants and a very positive experience during its total duration as well as for the future. Especially, the CAL network has been of decisive importance for the selection of two space missions focusing on TLEs. The "Atmosphere-Space Interactions Monitor (ASIM)", a instrument to be installed on the external pallet of the Columbus module on the International Space Station, has recently begun a Phase B study for the European Space Agency. Also, the French microsatellite project TARANIS (Tool for the Analysis of RAdiations from lightNIngs and Sprites) selected by the French space agency "Centre National d'Etudes Spatiales" is planned to be launched in 2011. The CAL network participants form the core science team of these missions and several of the CAL young scientists are expected also to become involved in the missions. In the spirit of the CAL project both ASIM and TARANIS will continue to contribute to our global understanding of sprites, jets and elves.

Acknowledgement. The CAL research training network was sponsored by the European Commission under the contract HPRN-CT-2002-00216. The CAL young scientists acknowledge all the senior scientists of the network for their support and thank M. Avngaard, G. Villa, A. Heilmann-Clausen, J. O. Hansen, K. Henriksen and T. H. Allin from the Danish National Space Center (Technical University of Denmark) for their involvement in the system set-up. They also acknowledge J.-M. Pichon from the "Observatoire de Physique du Globe de Clermont-Ferrand", and J.-M. Abbadie and all the technicians from the "Observatoire Midi-Pyrénées" for their help at the mountain tops.

Edited by: N. Crosby and M. Rycroft

Reviewed by: three anonymous referees

\section{References}

Allin, T. H., Neubert, T., and Laursen S.: Imaging systems in TLE research, in: Sprites, Elves and Intense Lightning Discharges, edited by: Füllekrug, M., Mareev, E. A., and Rycroft, M. J., Springer, NATO Science Series, 225, 101-121, 2006.

Allin, T. H.: Design Methods for High-Speed, High-resolution Digital Multispectral Imager, PhD Dissertation, Technical University of Denmark, 2005.

Arnold, N. F. and Neubert, T.: The Electric Earth : Cosmic Influences on the Atmosphere, Astron. Geophys., 43, 6.9-6.12, December 2002.

Crosby, N. B.: Enter the World of Sprites, Jets and Elves, Sci. Connect., 14 (French and Dutch version), December 2006.

Neubert, T.: On Sprites and their Exotic Kin, Science, 300, p. 747, 2003.

Neubert, T., Allin, T. H., Blanc, E., Farges, T., Haldoupis, C., Mika, Á., Soula, S., Knutsson, L., Van der Velde, O., Marshall, R. A., Inan, U., Sátori, G., Bór, J., Hughes, A., Collier, A., Laursen, S., and Lundgaard Rasmussen, I.: Co-ordinated observations of transient luminous events during the EuroSprite2003 campaign, J. Atmos. Sol.-Terr. Phys., 67, 807-820, 2005. 